\title{
THE GLOBULAR CLUSTER METALLICITY SCALE: EVIDENCE FROM STELLAR MODELS
}

\author{
Pierre Demarque, Christopher R. King, and Angeles Diaz \\ Yale University Observatory \\ Received 1981 December 21; accepted 1982 February 18
}

\begin{abstract}
Theoretical giant branches have been constructed to determine their relative positions for metallicities in the range $-2.3 \leq[\mathrm{Fe} / \mathrm{H}] \leq 0$. The models included the following improvements over previous studies: (1) in the convection zone, use of the variable mixing length formalism of Deupree and Varner in conjunction with Deupree's horizontally averaged opacity; (2) for the surface boundary conditions, a fit to the grid of model atmospheres of Bell et al.

A relation between $[\mathrm{Fe} / \mathrm{H}]$ and $(B-V)_{o, g}$ based on these models is presented which yields good agreement over the observed range of metallicities for galactic globular clusters and old disk clusters. The metallicity of 47 Tuc and M71 given by this calibration is about -0.8 dex.

Subject headings: clusters: globular - stars: abundances - stars: interiors
\end{abstract}

\section{INTRODUCTION}

It has long been recognized, ever since the work of Hoyle and Schwarzschild (1955), that metal content determines the relative positions of the giant branches of globular clusters and old disk clusters in the H-R diagram. Over the years, a number of methods based on this knowledge have been devised to rank old star clusters in the Galaxy according to their metallicities. But primarily because of our inability to model convective envelopes in a reliable way, stellar interior theory has failed to provide more than a qualitative check on the metallicity scale. The practice has been to adjust the parameter $\alpha$, the ratio of mixing length to pressure scale height in the convection zone so as to obtain agreement between theory and observation (see, e.g., Demarque and McClure 1977). The quantitative abundance scale has been calibrated on spectroscopically determined chemical abundances for individual stars. The work of Searle and Zinn (1978) and Zinn (1980) provides the most recent examples of this approach.

In recent years, the following advances have been made on several fronts which are relevant to this problem: (1) More reliable stellar atmosphere models for red giants of different metallicities (Bell et al. 1976; Gustafsson et al. 1975) have become available; (2) Infrared observations of individual giants in globular clusters have yielded, with the aid of model atmospheres, a more accurate effective temperature scale for these stars (Fogel, Persson, and Cohen 1981); (3) The use of echelle spectrographs on large reflectors has rendered possible abundance determinations of the atmospheres of globular cluster giants. So far these studies have confirmed previous metallicity estimates for the most metalpoor clusters (e.g., M92) but have led to confusion and controversy at higher metallicities (e.g., 47 Tuc and M71): (Cohen 1980; Cottrell and Da Costa 1981; Dickens, Bell, and Gustafsson 1979; Pilachowski, Canterna, and
Wallerstein 1980;Zinn 1981); (4) Recent studies of stellar convection based on two-dimensional (2D) hydrodynamic modeling have reduced the uncertainties in the use of the standard mixing length theory to describe convective stellar envelopes (Deupree 1979; Deupree and Varner 1980, hereafter referred to as DV).

In view of the above advances and in the hope of throwing some light on the current controversy on the abundances of the metal-rich globular clusters, we have reconsidered the problem from the point of view of stellar models, which is independent from echelle spectroscopy. Two improvements have been incorporated into our stellar evolution program: (1) the formalism of Deupree (1979) and DV to describe the structure of the convective envelope, and (2) the grid of nongray model atmospheres of Bell et al. (1976) for the surface boundary conditions.

With this approach, we have been able to derive the dependence of the giant-branch position in the H-R diagram on metallicity for the stars of low mass found in the globular clusters and the oldest disk clusters. Section II contains a description of the models and of the procedure used in constructing the giant branches. The results are discussed in $\S$ III and compared to previous calculations and to observation. Section IV summarizes the conclusions of the paper.

\section{IMPROVEMENTS IN THE PHYSICS}

\section{a) The Surface Convection Zone}

In the standard mixing length theory, the structure of the outer part of the convection zone and the radius of the models depend on the choice of the parameter $\alpha$ which is poorly determined. DV have described a modification to the mixing length theory based on $2 \mathrm{D}$ hydrodynamic models of stellar convective envelopes which decreases this uncertainty substantially. Their 
formulation sets the variation of $\alpha$ as a function of temperature in the outer layers of the convection zone.

According to DV, the effective value of $\alpha$ can be expressed in terms of temperature in the following way:

$$
\begin{aligned}
\log (\alpha) & =-2.85\left(T_{4}-0.7\right)+0.3 \quad & \text { for } T_{4} \leq 0.746 \\
& =6.36\left(T_{4}-0.97\right)^{2}-0.15 & \text { for } 0.746 \leq T_{4} \leq 1
\end{aligned}
$$

where $T_{4}$ is the temperature in units of $10^{4} \mathrm{~K}$. Because the $2 \mathrm{D}$ calculations could not be carried out beyond $10^{4} \mathrm{~K}$ due to spurious numerical effects at the lower boundary of the convection zone, one still needs to specify $\alpha$ at higher temperatures. The policy is to select a constant value $\alpha_{c}$, employ the analytic fit from the surface to the point at temperatures above $10^{4} \mathrm{~K}$ where this selected value is reached, and then use this constant $\alpha_{c}$ in all deeper layers.

This modification can be incorporated conveniently in stellar structure programs which use the standard mixing length theory. Equation (1) must be used in conjunction with a correction to the opacity designed to account for the effects of horizontal energy losses in the convective elements (Deupree 1979).

\section{b) The Surface Boundary Conditions}

The detailed structure of the outer atmospheric layers also affect appreciably the radii of red-giant models (Demarque, Laird, and VandenBerg 1981). The gray model atmospheres used in previous studies (Prather 1976; Sweigart and Gross 1978) were replaced by a grid of nongray model atmospheres for red giants due to Bell et al. (1976). In order to incorporate these simply as surface boundary conditions into the stellar evolution program, an analytic fit of the grid was made in the $\left(\log T_{\text {eff }}-[\mathrm{Fe} / \mathrm{H}]-\log g-\log P_{s}\right)$ space, where $P_{s}$ is the value of the pressure at the point in the atmosphere where the temperature $T=T_{\text {eff }}$.

The best fit, separated into two $T_{\text {eff }}$ ranges, produces values for $\log P_{s}$ accurate to within $5 \%$ in the worst case. The analytic equation can be written as:

$$
\log P_{s}=\sum_{i=1}^{12} C_{i} X_{i},
$$

where the coefficients $C_{i}$ and the variables $X_{i}$ are given in Table 1 . We note that the grid of atmospheres covers the range of metallicity of the interior models. In the case where $Y$ was varied, the effects of $Y$ on atmospheric structure were ignored. Small variations in $Y$ are unlikely to affect the results in a noticeable way (BöhmVitense 1979).

\section{c) The Grid of Models}

Because we are only concerned in improving the surface boundary conditions which do not affect the structure of the central regions of the models, and because the evolution of a low mass star along the giant branch is such a slow and time-consuming computation, complete evolutionary tracks were not constructed. Instead the position of the giant branches was

\begin{tabular}{|c|c|c|c|}
\hline & $T \leq 4500 \mathrm{~K}$ & $T \geq 4500 \mathrm{~K}$ & \\
\hline$i$ & $C_{i}$ & $C_{i}$ & $X_{i}$ \\
\hline 1 & +263.272120 & -326.591290 & 1 \\
\hline 2 & -142.616187 & +181.138924 & $\log T_{\text {eff }}$ \\
\hline 3. & -81.383119 & +16.862017 & $\log g$ \\
\hline 4. & -3.280648 & -8.346350 & {$[\mathrm{Fe} / \mathrm{H}]$} \\
\hline & +19.502037 & -24.912565 & $\left(\log T_{\text {eff }}\right)^{2}$ \\
\hline & -0.593038 & +0.180043 & $(\log g)^{2}$ \\
\hline & -0.047099 & -0.063132 & {$[\mathrm{Fe} / \mathrm{H}]^{2}$} \\
\hline 8 & +46.094303 & -9.799892 & $\left(\log T_{\text {eff }}\right)(\log g)$ \\
\hline 9 & +0.777299 & +2.175111 & $\left(\log T_{\mathrm{eff}}\right)([\mathrm{Fe} / \mathrm{H}])$ \\
\hline 10 & -0.012867 & -0.042264 & $(\log g)([\mathrm{Fe} / \mathrm{H}])$ \\
\hline & -6.472913 & +1.461345 & $\left(\log T_{\text {eff }}\right)^{2}(\log g)$ \\
\hline & +0.160034 & -0.051390 & $\left(\log T_{\text {eff }}\right)(\log g)^{2}$ \\
\hline
\end{tabular}

TABLE 1

Coefficients for Model Atmospheres Fit Equation (2)

determined by constructing stellar models at three luminosities (a horizontal-branch luminosity, a higher, and a lower luminosity) starting from existing models for giant stars. At each luminosity, each star was then evolved for five time steps (corresponding to about $10^{4}$ years for the brightest models and $4.9 \times 10^{6}$ years for the faintest, and in all cases to an increase in $\log L / L_{\odot}$ of about 0.01 ) in order to define more precisely the location of the new giant branch.

Table 2 contains the main grid of models. All models were constructed for a mass of $0.85 M_{\odot}$ and a helium abundance $Y=0.25$. The range of metallicities is $0.02 \geq Z \geq 0.0001$ by mass (or $0 \geq[\mathrm{Fe} / \mathrm{H}] \geq-2.3$ ). For the choice of $\alpha_{c}$, it seemed at first reasonable to use the value 1.4 based on a solar model constructed with the same program. This value, although questionable for the Sun in view of current uncertainties about the structure of the solar interior, was arbitrarily used for the main grid of giant models. The discussion in the next section shows that a value of 0.8 would have been more appropriate for $\alpha_{c}$.

In addition, Table 2 contains giant-branch positions determined for selected models with masses $M=0.70$ $M_{\odot}$ and $1.0 M_{\odot}$ and with helium abundances $Y=0.20$ and 0.30 in order to ascertain the effects of small variations in mass and helium content.

Except for the two major improvements just described, the calculations were performed with the help of the stellar evolution program developed by Prather (1976). The opacities were taken from Cox and Stewart (1970) and the nuclear energy generation rates from Fowler, Caughlan, and Zimmerman (1975). The description of physics was basically the same as that used in the redgiant models of Sweigart and Gross (1978).

\section{RESULTS AND DISCUSSION}

\section{a) Comparison with Standard Models}

In order to compare the models described in the previous section to models constructed with standard physical assumptions, we also calculated the effects of metallicity on the giant branch with the same stellar 
TABLE 2

Grid OF Giant MODELS

\begin{tabular}{|c|c|c|c|c|}
\hline$M$ & $Y$ & $L^{\mathrm{a}}$ & $\log T_{\text {eff }}$ & $\log L / L_{\odot}$ \\
\hline \multicolumn{5}{|c|}{$Z=0.02,[\mathrm{Fe} / \mathrm{H}]=0$} \\
\hline 0.85 . & 0.25 & I & 3.65979 & 1.3308 \\
\hline $0.85 \ldots$ & 0.25 & II & 3.64697 & 1.6818 \\
\hline \multicolumn{5}{|c|}{$Z=0.01,[\mathrm{Fe} / \mathrm{H}]=-0.3$} \\
\hline 0.85 . & 0.25 & I & 3.67612 & 1.3272 \\
\hline 0.85 . & 0.25 & II & 3.66427 & 1.6581 \\
\hline 0.85 . & 0.25 & III & 3.58420 & 2.9787 \\
\hline 1.0 . & 0.25 & II & 3.64306 & 2.1993 \\
\hline 0.7 & 0.25 & II & 3.67897 & 1.0237 \\
\hline 0.85 & 0.2 & II & 3.66275 & 1.5794 \\
\hline 0.85 & 0.3 & II & 3.66545 & 1.7412 \\
\hline \multicolumn{5}{|c|}{$Z=0.004,[\mathrm{Fe} / \mathrm{H}]=-0.7$} \\
\hline 0.85 & 0.25 & I & 3.69339 & 1.3081 \\
\hline 0.85 & 0.25 & II & 3.68354 & 1.6244 \\
\hline 0.85 . & 0.25 & III & 3.61093 & 2.9285 \\
\hline \multicolumn{5}{|c|}{$Z=0.001,[\mathrm{Fe} / \mathrm{H}]=-1.3$} \\
\hline 0.85 & 0.25 & I & 3.71471 & 1.2527 \\
\hline 0.85 & 0.25 & II & 3.70601 & 1.5523 \\
\hline $0.85 \ldots$ & 0.25 & III & 3.64408 & 2.8318 \\
\hline \multicolumn{5}{|c|}{$Z=0.0004,[\mathrm{Fe} / \mathrm{H}]=-1.7$} \\
\hline 0.85 & 0.25 & I & 3.72618 & 1.2075 \\
\hline 0.85 & 0.25 & II & 3.71755 & 1.5046 \\
\hline 0.85 . & 0.25 & III & 3.66265 & 2.7676 \\
\hline 1.0 & 0.25 & II & 3.70370 & 1.9917 \\
\hline 0.7 & 0.25 & II & 3.72852 & 0.9481 \\
\hline 0.85 & 0.2 & II & 3.71793 & 1.4230 \\
\hline 0.85 . & 0.3 & II & 3.71831 & 1.5787 \\
\hline \multicolumn{5}{|c|}{$Z=0.0001,[\mathrm{Fe} / \mathrm{H}]=-2.3$} \\
\hline 0 & 0.25 & II & 3.72900 & 1.4207 \\
\hline 0.85 & 0.25 & III & 3.68029 & 2.6667 \\
\hline
\end{tabular}

${ }^{\text {a }} L$ denotes a luminosity level: I. Fainter than HB luminosity; II. HB luminosity; III. Brighter than HB luminosity.

evolution program, but using the standard mixing length theory and the Eddington approximation in the surface radiative layers. The calculations were performed with a constant $\alpha=1$, and the results are shown in Table 3 . These models represent one point on each evolutionary track and, assuming the same slope as Sweigart and Gross (1978), one can compare the spread in $T_{\text {eff }}$ over the range of metallicities. We note a systematic shift in $T_{\text {eff }}$ from the standard to the DV models which will be discussed in the next paragraph. We also note that in the same metallicity interval $-2.3 \leq[\mathrm{Fe} / \mathrm{H}] \leq-0.3$, the spread in $T_{\text {eff }}$ is smaller for the DV models. We shall see later that this difference improves the comparison of the position of the M67 giant branch to that of the globular clusters.

The dependence of $T_{\text {eff }}$ on $\alpha$ is illustrated in Figure 1 for both the standard and the DV models. The
TABLE 3

STANDARD Giants

\begin{tabular}{cccc}
\hline \hline$Y$ & \multicolumn{1}{c}{$Z$} & \multicolumn{1}{c}{$\log T_{\text {eff }}$} & $\log L / L_{\odot}$ \\
\hline $0.25 \ldots \ldots \ldots \ldots$ & 0.02 & 3.60098 & 1.6586 \\
$0.25 \ldots \ldots \ldots \ldots$ & 0.01 & 3.62171 & 1.6257 \\
$0.25 \ldots \ldots \ldots \ldots$ & 0.004 & 3.64308 & 1.5929 \\
$0.25 \ldots \ldots \ldots \ldots$ & 0.001 & 3.67389 & 1.5267 \\
$0.25 \ldots \ldots \ldots \ldots$ & 0.0004 & 3.68192 & 1.4736 \\
$0.25 \ldots \ldots \ldots \ldots$ & 0.0001 & 3.69362 & 1.3933 \\
\hline $0.30 \ldots \ldots \ldots \ldots$ & 0.001 & 3.67796 & 1.6020 \\
$0.20 \ldots \ldots \ldots \ldots$ & 0.001 & 3.67532 & 1.4465 \\
\hline
\end{tabular}

comparison has been carried out for the composition parameters $(X, Z)=(0.749,0.001)$ and for $0.5 \leq \alpha \leq 2.0$. Our calculations confirm the conclusions of DV that their description of convection lowers the sensitivity of $T_{\text {eff }}$ on the choice of $\alpha$. As seen in Figure 1, this is particularly true for the range $1.0 \leq \alpha \leq 2.0$ which they investigated. There remains, however, an uncertainty in $\alpha$ at temperatures above $10^{4} \mathrm{~K}$ in the DV models, which is the cause of the dependence of $T_{\text {eff }}$ on $\alpha$ shown in Figure 1. Detailed hydrodynamical modeling to temperatures higher than $10^{4} \mathrm{~K}$ in the convective envelope would remove this formal uncertainty in $\alpha$ and is badly needed. In the absence of such models, we shall be allowed shifts in $\log T_{\text {eff }}$ in the theoretical H-R diagram, although only half as large as in the standard case for the same variation in $\alpha$.

In the comparison of our model giant branches with observation, we shall find that best agreement is obtained when $\alpha_{c}$ is chosen near 0.8 , a seemingly reasonable value since it is near the value of $\alpha$ given by equation (1) in the vicinity of $10^{4} \mathrm{~K}^{1}$

\footnotetext{
${ }^{1}$ Dr. Deupree has kindly permitted us to quote from a recent letter: "From the great range in values and the uncertainties involved at temperatures above $10,000 \mathrm{~K}$, I would say that just about any value between 0.4 and 1.5 for the ratio of mixing length to pressure scale height could be supported, although values in the middle of this range (including 0.8 ) are, I should think, more probable."
}

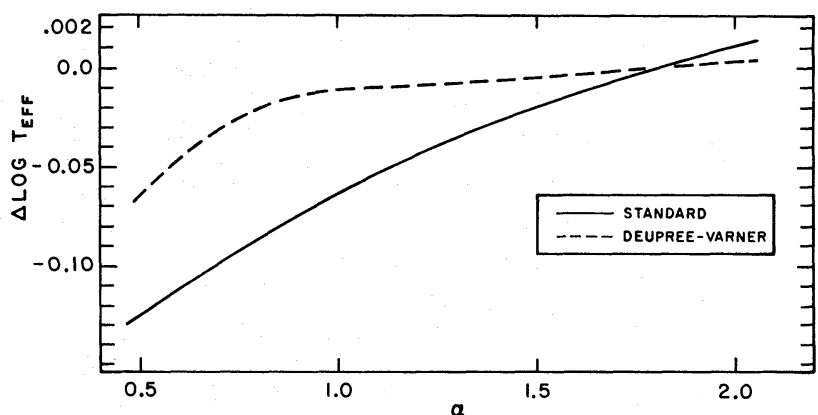

FIG. 1.-Comparison of mixing-length schemes. For the DeupreeVarner variable mixing length scheme, the value of $\alpha$ is that of the constant $\alpha_{c}$ (defined in the text). The values of $T_{\text {eff }}$ are read off at a horizontal branch luminosity level. 


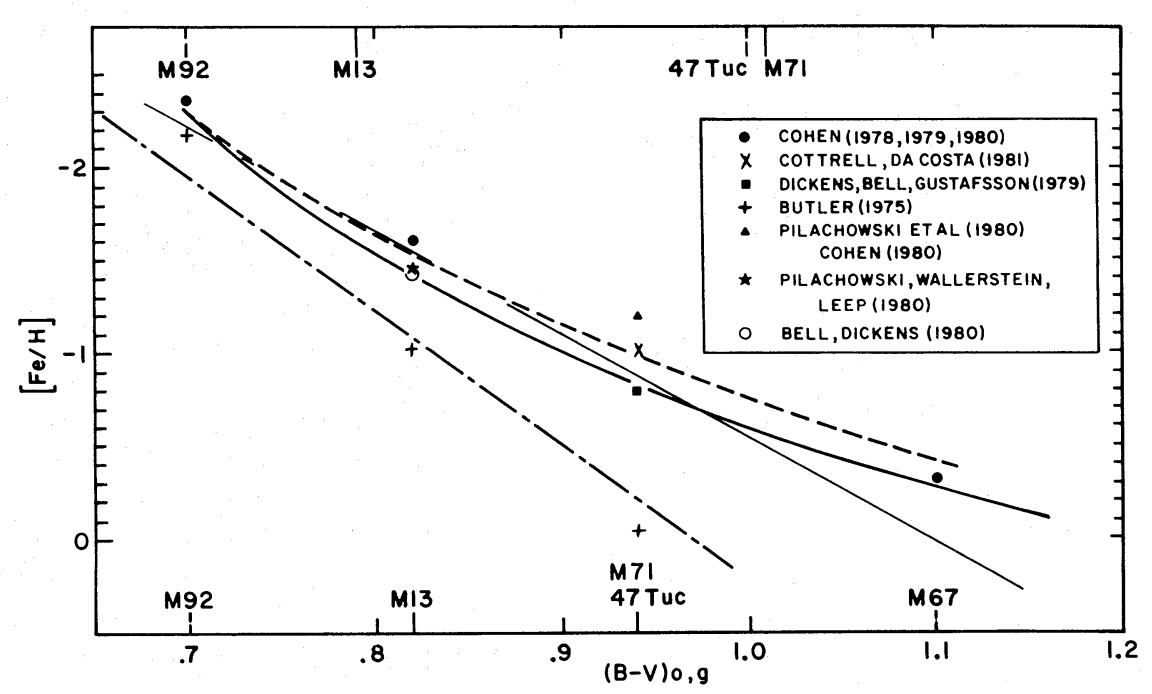

FIG. 2.-Globular cluster metallicity scale. The solid curve represents our calibration. For comparison the same procedure was followed to construct a calibration curve using the models of Sweigart and Gross (1978) for $\left(0.70 M_{\odot}, Y=0.30\right)$ (dashed curve). The dot-dashed line is Butler's (1975) calibration based on the metallicity of RR Lyrae variables in the clusters given by the $\Delta S$ index. The solid straight line shows Sandage's (1981) new calibration. The $(B-V)_{o, g}$ colors of M92, M13, M71, and 47 Tuc are taken from Butler (lower scale) and from Sandage (upper scale). The color of the M67 giant branch is from the work of Racine (1971). The metallicities of M92, M13, M71, 47 Tuc, and M67 measured by several authors are marked on the diagram using different symbols.

\section{b) Comparison with Observation}

In Figure 2, we have constructed a theoretically calibrated $(B-V)_{o, g}$ versus $[\mathrm{Fe} / \mathrm{H}]$ relation for comparison with observation. This calibration was obtained in the following way: From the theoretical giant branches, the value of $\log T_{\text {eff }}$ was read at $\log L / L_{\odot}=$ 1.70 , chosen as the horizontal-branch luminosity, and converted to $(B-V)_{o, g}$ by interpolation in the model atmosphere tables of Bell and Gustafsson (1978). We fixed $\alpha_{c}$ by requiring agreement in the $[\mathrm{Fe} / \mathrm{H}]$ versus $(B-V)_{o, g}$-plane between the position of the giant branch of M92 given by Frogel, Persson, and Cohen (1981) and the giant-branch models for $[\mathrm{Fe} / \mathrm{H}]=-2.3$ dex. The cluster M92 was chosen because its metallicity is relatively well determined among globular clusters. Agreement was achieved by applying a shift toward lower temperatures of $\Delta \log T_{\text {eff }}=0.02$ to the models of Table 2, corresponding to a choice of $\alpha_{c} \sim 0.8$. Note that the curve passes through the position of M13 given by Bell and Dickens (1980) and Pilachowski, Wallerstein, and Leep (1980). This also yields agreement with the M67 giant branch for $[\mathrm{Fe} / \mathrm{H}] \sim-0.3$ dex a metallicity consistent with spectroscopic estimates of -0.3 dex by Griffin (1975) and -0.4 dex by Cohen (1980). From Table 2, we calculate that an increase in the giant masses from $0.85 M_{\odot}$ to $1 M_{\odot}$ (a more likely mass for M67 giants) corresponds to an increase in $\log T_{\text {eff }}$ of about 0.005 . In view of the uncertainties in the distance modulus of the cluster which introduce errors of \pm 0.005 in $\log T_{\text {eff }}$ and additional errors of the same magnitude in the temperature scale derived from the infrared data of Frogel, Persson, and Cohen (1981), the fit for M67 seems satisfactory.
Finally, we wish to emphasize that in constructing our models we have assumed a solar distribution of the relative abundances of chemical elements with low ionization potentials. It is possible that variations in the abundances of the major electron donors, which contribute to the continuous opacity in the atmosphere through $\mathrm{H}^{-}$dissociation, would modify our results. The available observational evidence, however, suggests that the relative abundance of low ionization potential elements vary little from solar in globular clusters (Cohen 1980).

\section{CONCLUSIONS}

We have found that the use of DV's more realistic treatment of convection, combined with the use of model atmospheres for the surface boundary conditions, reduces substantially the uncertainties in the effective temperatures of red-giant models.

The present models provide an adequate representation of the observed globular clusters giant branches and yield good agreement in the H-R diagram with both the very metal-poor cluster M92 and the old disk cluster M67. Since these clusters are found near the extremes of the metallicity range observed in old star clusters, it is not unreasonable to assume the validity of the calibration for intermediate metallicities. Using this same calibration for 47 Tuc and M71 we find an internally consistent value near $-0.8 \mathrm{dex}$, in excellent agreement with the estimate of Dickens, Bell, and Gustafsson (1979) for 47 Tuc, and significantly more metal-rich than found by Cohen (1980) and Pilachowski, Canterna, and Wallerstein (1980). 
We conclude that if one is to accept the uniformity of the relative abundances of heavy elements in clusters of varying metallicities, the evidence from stellar models suggests a one-to-one correspondence between the color of the giant branch and metallicity.
The authors with to acknowledge the assistance of Donna Keith in the early phases of this work and helpful comments from G. Da Costa on the first draft of this paper. Partial support from grant AST 80-23745 from the National Science Foundation is gratefully acknowledged.

\section{REFERENCES}

Bell, R. A., and Dickens, R. J. 1980, Ap. J., 242, 657.

Bell, R. A., and Gustafsson, B. 1978, Astr. Ap. Suppl., 34, 229.

Bell, R. A., Gustafsson, B., Eriksson, K., and Nordlund, A. 1976, Astr. Ap. Suppl., 23, 37.

Butler, D. 1975, Pub.A.S.P., 87, 559.

Böhm-Vitense, E. 1979, Ap. J., 234, 521.

Cohen, J. G. 1978, Ap. J., 223, 487. . 1979, Ap. J., 231, 751. 1980, Ap. J., 241, 981.

Cottrell, P. J., and Da Costa, G. S. 1981, Ap. J. (Letters), 245, L78.

Cox, A. N., and Stewart, J. N. 1970, Ap. J. Suppl., 19, 261.

Demarque, P., Laird, J. B., and VandenBerg, D. A. 1981, in $I A U$ Colloquium 68, Schenectady, NY, Astrophysical Parameters for Globular Clusters, ed. A. G. D. Philip and D. S. Hayes, Dudley Obs. Rept. 15, p. 45.

Demarque, P., and McClure, R. D. 1977, Ap. J., 213, 716.

Deupree, R. G. 1979, Ap. J., 234, 228.

Deupree, R. G., and Varner, T. M. 1980, Ap. J., 237, 558 (DV).

Dickens, R. J., Bell, R. A., and Gustafsson, B. 1979, Ap. J., 232, 428.

Fowler, W. A., Caughlan, G. R., and Zimmerman, B. A. 1975, Ann. Rev. Astr. Ap., 13, 69.
Frogel, J. A., Persson, S. E., and Cohen, J. G. 1981, Ap. J., 246, 842. Griffin, R. 1975, M.N.R.A.S., 171, 181.

Gustafsson, B., Bell, R. A., Eriksson, K., and Nordlund, A. 1975, Astr. Ap., 42, 407.

Hoyle, F., and Schwarzschild, M. 1955, Ap. J. Suppl., $2,1$.

Pilachowski, C. A., Canterna, R., and Wallerstein, G. 1980, Ap. J. (Letters), 235, L21.

Pilachowski, C. A., Wallerstein, G., and Leep, E. M. 1980, Ap. J., 236, 508.

Prather, M. 1976, Ph.D. thesis, Yale University.

Racine, R. 1971, Ap. J., 168, 393.

Sandage, A. 1981, preprint.

Searle, L., and Zinn, R. 1978, Ap. J., 225, 357.

Sweigart, A. V., and Gross, P. G. 1978, Ap. J. Suppl., 36, 405.

Zinn, R. 1980, Ap. J. Suppl., $42,19$.

1981, in IAU Colloquium 68, Schenectady, NY, Astrophysical Parameters for Globular Clusters, ed. A. G. D. Philip and D. S. Hayes, Dudley Obs. Rept. 15, p. 45.

P. Demarque and C. R. King: Yale University Observatory, Box 6666, New Haven, CT 06511

A. DiAz: Royal Greenwich Observatory, Herstmonceux Castle, Hailsham, Sussex, U.K. 